\title{
Avaliação Bioquímica do líquido cefalorraquidiano de cães normais e de cães jovens portadores de encefalite por cinomose.
}

\author{
Biochemical evaluation of the cerebrospinal fluid from \\ clinically normal dogs and from young dogs with distemper \\ enchephalitis.
}

CORAESPONDENCE TO Mary Marcondes Fertosa Curso de Medicina Veterinária de UNESP

Aug Clóvis Pestana, 793 16050-680 - Aracatuba - SP - Brasil

1 - Curso de Medicina Veterinária da UNESP Araçatuba - SP 2 - Faculdade de Medicina Veterinária e Zootecnia da UNESP - Botucatu - SP

\author{
Mary Marcondes FEITOSA', Aguemi KOHAYAGAWA²; Francisco Leydson Formiga FEITOSA²; \\ Paulo Roberto CURI'2; Suely Regina Kato MOGAMI²
}

\section{RESUMO}

O presente experimento teve como objetivos estabelecer padrões de referência para constituintes do liquido cefalorraquidiano, determinar os valores de cloretos, creatina fosfoquinase e aspartato aminotransferase no soro sangüineo e dosagem de glicose plasmática, na tentativa de verificar se existe correlação entre essas variáveis no sangue e no líquor, de cães normais e de cães jovens portadores de encefalite por cinomose. Foram utilizados 40 animais, sendo 20 cães normais e 20 cães com cinomose. 0 exame bioquimico do líquor dos cães normais permitiu o estabelecimento de valores para concentração de glicose: $86,09 \pm 17,76 \mathrm{mg} / \mathrm{dl}$, niveis de cloretos: $117,36 \pm 21,35 \mathrm{mEq} / \mathrm{L}$, niveis de creatina fosfoquinase; 3,77 $\pm 1,72 \mathrm{UI} / \mathrm{L}$ e níveis de aspartato aminotransferase $7,43 \pm 4,61 \mathrm{UI} / \mathrm{L}$. $\mathrm{O}$ exame bioquímico do líquor de cães com cinomose permitiu o estabelecimento de valores para concentração de glicose: $80,85 \pm$ $14,82 \mathrm{mg} / \mathrm{dl}$, niveis de cloretos: $118,67 \pm 12,82 \mathrm{mEq} / \mathrm{L}$, niveis de creatina fosfoquinase: $26,38 \pm 29,27 \mathrm{UI} / \mathrm{L}$ e níveis de aspartato aminotransferase; $65,70 \pm 23,72 \mathrm{UI} / \mathrm{L}$. Os niveis de cloretos, creatina fosfoquinase e aspartato aminotransferase no soro, e glicose no plasma nos cães normais e nos cães com cinomose foram, respectivamente, 103,43 $\pm 16,00$ e 103,43 $\pm 11,99 \mathrm{mEq} / \mathrm{L}, 95,75 \pm 22,83$ e $272,50 \pm 140,95 \mathrm{UI} / \mathrm{L}, 23,77 \pm 5,20$ e 23,44 \pm 4,81 UI/L, 119,12 $\pm 21,46$ e 106,12 \pm 17,73 mg/dl. Verificou-se uma correlação positiva entre a concentração de glicose liquórica e plasmática nos animais normais e nos animais com cinomose, e entre os niveis de CPK liquórica e sérica nos animais com cinomose. Não foram constatadas diferenças estatisticamente significativas entre os dois grupos de animais com relação aos niveis de cloretos do líquor e do soro. As amostras do líquor dos cães com cinomose apresentaram valores mais elevados de CPK e AST do que as amostras dos cães normais.

UNITERMOS: Líquido cerebioespinhal; cães; cinomose; sistema nervoso.

\section{INTRODUÇÃO}

$\mathrm{O}$ líquido cefalorraquidiano (LCR) é um fluido aquo-so e incolor que ocupa o espaço subaracnóide, os ventrículos cerebrais e o canal central da medula e, segundo Kay et al. ${ }^{18}$ (1974), Benjamin'2 (1978) e Reis et al. ${ }^{24}$ (1980), possui as funções de proteção do sistema nervoso contra agentes infecciosos, permitindo a distribuição mais ou menos homogênea de elementos de defesa como leucócitos e anticorpos, e de agente de troca de metabólitos entre o sangue e o cérebro ${ }^{21,24}$. Greene ${ }^{13}$ (1984) afirma que a análise do líquido cefalorraquidiano é de grande importância na avaliação de doenças neurológicas, particularmente aquelas de origem infecciosa, pois sua composição pode variar diretamente com processos patológicos do sistema nervoso central ${ }^{13}$. O presente trabalho teve como objetivos. 1) Estabelecer padrōes de referência para constituintes do líquido cefalorraquidiano de cães normais e de cães portadores de encefalite por cinomose, determinados pelo exame bioquímico (cloretos, glicosc, creatina fosfoquinase e aspartato aminotransferase). 2) Determinar (os valores de cloretos, creatina fosfoquinase e aspartato aminotransferase no soro sangüíneo, bem como a dosagem de glicose plasmática, na tentativa de verificar se existe cortelação entre esses parâmetros. no sangue e no líquor, de cães normais e de cães com cinomose.

\section{MATERIAL E MÉTODOS}

No presente trabalho foram utilizados dois grupos de animais. O primeiro grupo constou de 20 cães, 12 machos e 8 fêmeas, sem raça definida, entre seis e 48 meses de idade, clinicamente sadios. O segundo grupo era constituído de 20 cães, 15 machos e 5 fêmeas, sem raça definida. com idade máxima de 18 meses, portadores de encefalite por cinomose, encaminhados ao ambulatório do Hospital Veterinário da Faculdade de Medicina Veterinária e Zootecnia - UNESP - Campus de Botucatu. As amostras de líquido cefalorraquidiano foram obtidas através de punção da cisterna magna. Além do líquor. foram colhidas as seguintes amostras de cada animal: 1) $5 \mathrm{ml}$ de sangue total com anticoagulante Fluoreto de Sódio a $10 \%$ para a dosagem de glicose. 2) $10 \mathrm{ml}$ de sangue total para a obtenção de soro e determinação de cloretos, creatina fosfoquinase e 


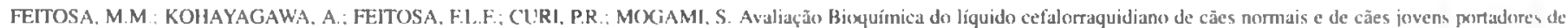
encefalite por cinomox. Braz. J. vet. Res. anim. Sci. São Paulo, v. 34. n.2. p.99-102. 1997.

aspartato aminotransferase. A Glicose Liquórica e Plasmática foi deterninada pelo método Kit Glicose E Reactoclin da CELM. Os níveis de Cloretos L iquóricos e Séricos foram delerminados pelo método Kit Cloro Colorimétrico Reactoclin da CELM. A Creatina Fosfoquinase Liquórica e Sérica foi determinada através do métoxdo Kit CK-NAC Reactoclin da CELM. A Aspartato Aminotransferase Liquórica e Sérica foi determinada através do método Kit AST (GOT) Reactoclin da CELM. Os dados obtidos em cada análise foram tabulados e submetidos à análise estatística para a comparação das variáveis. As comparaçöes entre os grupos G1 e G2 foram efetuadas através do teste t para duas amostras independentes (Zar" ${ }^{31}$ 1984). As estatísticas calculadas foram consideradas significativas quando $\mathrm{p}<0,05^{3 !}$.

\section{RESULTADOS}

Os valores para concentração de glicose, níveis de cloretos. niveis de creatina fosfoquinase e níveis de aspartato aminotransferase, no líquor e no soro, acham-se resumidos nas Tabs. 1 e 2, respectivamente. As inter-relações entre os pares de variáveis do líquor e do soro encontram-se na Tab. 3

TABELA 1

Valores Médios* da concentraçāo de glicose, niveis de cloretos, níveis de creatina fosfoquinase (CPK) e aspartato aminotransferase (AST) do líquor (LCR) de cāes normais (G1) e de cāes com cinomose (G2), bem como a apresentaçāo do cálculo e comentário estatístico. Botucatu, 1992.

\begin{tabular}{c|c|c|c|c} 
Variável & Cães normais (G1) & $\begin{array}{c}\text { Cães c/ Cinomose } \\
(\mathrm{G} 2)\end{array}$ & $\begin{array}{c}\text { Cálculo } \\
\text { Estatístico }\end{array}$ & $\begin{array}{c}\text { Comentário } \\
\text { Estatístico }\end{array}$ \\
\hline Glicose (mg/dl) & $86,09 \pm 17,76$ & $80,85 \pm 14,82$ & $\mathrm{t}=1,013 ; p<0,30$ & $\mathrm{G} 1=\mathrm{G} 2$ \\
Cloretos (mEq/L) & $117,36 \pm 21,35$ & $118,67 \pm 12,82$ & $\mathrm{t}=0,234 ; \mathrm{p}>0,50$ & $\mathrm{G} 1=\mathrm{G} 2$ \\
CPK (UI/L) & $3,77 \pm 1,72$ & $26,38 \pm 29,27$ & $\mathrm{t}=3,448 ; p<0,001$ & $\mathrm{G} 1<\mathrm{G} 2$ \\
AST (UI/L) & $7,43 \pm 4,61$ & $65,70 \pm 23,72$ & $\mathrm{t}=10,785 ; p<0,001$ & $\mathrm{G} 1<\mathrm{G} 2$ \\
\hline
\end{tabular}

"Dados expressos em média $(x) \pm$ desvio padrão (DP)

TABELA 2

Valores Médios" da concentraçāo de glicose do plasma, e níveis de cloretos, niveis de creatina fosfoquinase, (CPK) e aminotransferase (AST) do soro de cães normais (G1) e de cāes com cinomose (G2), bem como a apresentaçāo do cálculo e comentário estatístico. Botucału, 1992.

\begin{tabular}{c|c|c|c|c} 
Variável & Cães normais (G1) & $\begin{array}{c}\text { Cães c/ Cinomose } \\
(\mathrm{G} 2)\end{array}$ & $\begin{array}{c}\text { Cálculo } \\
\text { Estatístico }\end{array}$ & $\begin{array}{c}\text { Comentário } \\
\text { Estatístico }\end{array}$ \\
\hline Glicose (mg/dl) & $119,12 \pm 21,46$ & $106,12 \pm 17,73$ & $\mathrm{t}=2,088 ; p<0,05$ & $\mathrm{G} 1>\mathrm{G} 2$ \\
Cloretos (mEq/L) & $103,43 \pm 16,00$ & $103,43 \pm 11,99$ & $\mathrm{t}=0,001 ; p>0,50$ & $\mathrm{G} 1=\mathrm{G} 2$ \\
CPK (UI/L) & $95,75 \pm 22,83$ & $272,50 \pm 140,95$ & $\mathrm{t}=5,536 ; \mathrm{p}>0,001$ & $\mathrm{G} 1<\mathrm{G} 2$ \\
AST (UI/L) & $23,77 \pm 5,20$ & $23,44 \pm 4,81$ & $\mathrm{t}=0,212 ; \mathrm{p}>0,50$ & $\mathrm{G} 1=\mathrm{G} 2$ \\
\hline
\end{tabular}

*Dados expressos em média $(x) \pm$ desvio padrāo (DP)

TABELA 3

inter-relaçōes entre pares de variáveis do liquor $\Theta$ do soro dos cōes normais (G1) e dos cāes com cinomose (G2). Coeficiente de correlaçāo linear e nivel de significância.

\begin{tabular}{c|c|c|c} 
Grupo & Variáveis & Coeficiente correlação & Nível significância \\
\hline G1 & Glicose LCR $\times$ Glicose Plasma & $r=+0,85$ & $P>0,01$ \\
G2 & Glicose LCR $\times$ Glicose Plasma & $r=+0,83$ & $P>0,01$ \\
G2 & CPK LCR $\times$ CPK Soro & $r=+0,86$ & $P>0,01$
\end{tabular}

\section{DISCUSSĀO}

Os valores obtidos para a concentração de glicose no liquor de cães normais foram superiores aos descritos por Coles ${ }^{5}(1980) \mathrm{e}$ Femandes ${ }^{12}$ (199()), mas coincidiram com os de Vogel et al. ${ }^{27}$ (1953), Benjamin' (1978), Kirk'14 (1984) e Feldman" (1989), com exceção de um animal que apresentou uma concentração liquórica um pouco clevada. provavelmente como conseqüência da concentração plasmática no momento da determinação, yue também foi a mais alta de todas as amostras ${ }^{2.5 .11,12,19,27}$. Nos cães com cinomose, os níveis sćricos foram menores do que nos cães normais, devido provavelmente à anorexia manifestada por aqueles. De acordo com as recomendaçōes de Cook: Denicolat (1988), os níveis de glicose sćrica foram dosados no momento da colheita do líquors. Em indivíduos nomais há uma relação constantc entre as taxas de glicose do líquor e do sangue. Nos animais nomais e nos animais com cinomose houve uma correlação positiva entre a glicose liquórica e sérica, sendo que a glicose liquórica variou de $6.3 \%$ a $85 \%$ dos niveis sćricos, concondando com as informaçōes de 


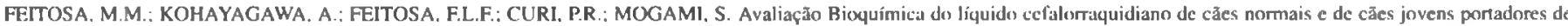
encefalite por cinomosc. Braz. J. vet. Res. anim. Sci., São Paulo, v.34, n.2, p.99-102, 1997.

\section{SUMMARY}

Cerebrospinal fluid samples were obtained from 20 normal dogs and from 20 dogs with canine distemper encephalitis, for biochemical evaluation. Blood samples were taken simultaneously and the values of chlorides, glucose, creatine phosphokinase and glutamic oxaloacetic transaminase were determined to verify if there is any correlation between those constituents from blood and from cerebrospinal fluid. Biochemical evaluation of the cerebrospinal fluid from normal dogs were: glucose concentration: $86,09 \pm 17,76 \mathrm{mg} /$ dl, chloride levels: $117,36 \pm 21,35 \mathrm{mEq} / \mathrm{L}$, creatine phosphokinase levels: $3.77 \pm 1.72 \mathrm{UI} / \mathrm{L}$ and glutamic oxaloacetic levels: $7.43 \pm 4.61$ UI/L. Biochemical evaluation of the cerebrospinal fluid from dogs with distemper encephalitis were: glucose concentration: $88.85 \pm$ $14.82 \mathrm{mg} / \mathrm{dl}$, chloride levels: $118.67 \pm 12.82 \mathrm{mEq} / \mathrm{L}$, creatine phosphokinase levels: $26.38 \pm 29.27 \mathrm{UI} / \mathrm{L}$ and glutamic oxaloacetic levels: $65.70 \pm 23.72 \mathrm{UI} / \mathrm{L}$. Chloride, creatine phosphokinase and glutamic oxaloacetic seric levels and glucose plasmatic concentration in normal dogs and in dogs with distemper encephalitis were, respectively, $103.43 \pm 16.00$ and $103.43 \pm 11,99 \mathrm{mEq} / \mathrm{L}, 95.75 \pm 22.83$ and $272.50 \pm 140.95 \mathrm{UI} / \mathrm{L}, 23.77 \pm 5.20$ and $23.44 \pm 4.81 \mathrm{UI} / \mathrm{L}, 119.12 \pm 21.46$ and $106.12 \pm 17.73 \mathrm{mg} / \mathrm{dl}$. There was a positive correlation between the glucose plasmatic concentration and the glucose concentration in cerebrospinal fluid in normal dogs and in dogs with distemper encephalitis, and between creatine phosphokinase levels in cerebrospinal fluid and seric levels in dogs with distemper. There were no differences between chloride seric level and chloride in cerebrospinal fluid in normal dogs and in dogs with distemper. CPK and GOT levels in cerebrospinal fluid from dogs with distemper encephalitis were higher than in normal dogs.

UNITERMS: Cerebrospinal fluid; Dogs; Distemper; Nervous system.

\section{REFERÊNCIAS BIBLIOGRÁFICAS}

I-BELTON, N.R.; BACKUS, R.E.; MILLICHAP, J.G. Serum creatine phosphokinase activity in epilepsy. Neurology, Cleveland v.17, n. , p. 1073, 1967.

2-BENJAMIN. M. Cerebrospinal Fluid Examination. In: Outline of veterinary clinical pathology. Ames, Iowa State University Press, 1978. p. 276-85.

3-CARDINET, G.H. Skeletal muscle function In: KANEKO, J.J. Clinical biochemistry of domestic animals. San Diego, Academic Press. 1989, p.462-95.

4-CHRISMAN. C.L. Averill Jr. D.R. Diseases of peripheral nerves and muscle. In: EI TINGER. S.J. Textbook of veterinary internal medicine. Philadelphia, W.B. Saunders. 1983, p.608-53.

5-COLES, E.H. Veterinary clinical pathology. Philadelphia; W.b. Saunders, 1980, p. 362-76: Cerebrospinal fluid.

6-COOK. J.R.; DENICOLA. D.B. Cerebrospinal fluid. Veterinary Clinics of North America Small Animal Practice. Philadelphia, v.18, n.3, p.475-99. 1988.

7-CROFT, P.G. Biochemistry of the cercbro-spinal fluid of the dog. Veterinary Record. London, v.67, n.47, p.872-6, 1955

8-DELAHUNTA A. Cerebrospinal fluid and hydrocephalus. In: Veterinary Neumatomy and Clinical Neumlogy. Philadelphia, W.B. Saunders, 1977, p. 33.56.

9-DOXEY, D.I.. Patologia clínica e métodos de diagnóstico. Rio de Janeiro, Interamericana, 1985. 306p.

10-DUNCAN, J.R.; OLIVER J.R.: J.E.; MAYHEW, I.G. Laboratory examinations. In: OLIVER, J.E.; HOERLEIN, B.F.; MAYHEW. I.G. Veterinary neurology. Philadelphia. W.B. Saunders, 1987, p.57-64.

11-FEI.DMAN, B.F. Cerebrospinal fluid. In: KANEKO, J.H. Clinical biochemistry of domestic animaks. San Diego. Academic Press, 1989, p.835-65.

12-FERNANDES, W.R. Determinação dos valores liquóricos normais de glicose, proteína. globulina. uréia, creatina fosfoquinase (CK), aspartato aminotranferase (AST), leucócitos e da coloração, turbidez e coagulabilidade em cães sadios. Brazilian ,Journal of Veterinary Research and Anintal Science, São Paulo. v.27, n.2, p.209-16, 1990).

13-GREENE, C.E. Infections of the central nervous system. In (Clinical microbiolog and infectious discases of the dogn and cat, Philadelphia W.B.Saunders, 1984. p.287-93.

14-HIBBS, C.M. COLES, E.H. Transaminases in blood, urine and cenebrospinal lluid of nomual and unilaterally nephrectomized dogs. Proceedings of the Society for Experimental Biology, New York, v.118, p. 1059-62, 1965

15-INDRIERI. R.J.; HOI.I.IDAY. T.A.; KFEN. C.L. Critical evaluation of creatine phosphokinase in cerebrospinal fluid of dogs with neurologic disease. American Journal of Veterinary Research, Schaumburg. v.41, n.8. p. 1299-303.1980.

16-JORDAN, J.E. Nomal laboratory values in beagles dogs of twelve to eighteen mounths of age. Schaumburg, v.38, n.4. p.509-13,1977.
17-KANEKO, J.J. Clinical biochemistry of domestic animals. San Diego. Academic Press, 1989. 932p.

18-KAY, WJ.; ISRAEI., E.; PRATA, R. Cerebrospinal fluid. Veterinary Clinics of North America Small Animal Practice, Philadelphia. v.4, n.2, p.419-35, 1974.

19-KIRK. R.W. Atualizałão terapêutica veterinária. São Paulo, Manole, 1984, 1495p.

20-LENDING, M.; SLOBODY, L.B.; MESTERN, J. Effect of convulsions on cerebrospinal fluid and plasma activity of glutamic oxalacetic transaminase and lactic dehydrogenasc. Neurology, Cleveland, v.9. p.672-7. 1959.

21-MAYHEW, 1.G., BEAL, C.R. Techniques of analysis of cerebrospinal nuid. Veterinary Clinics of North America Small Animal Practice, Philactelphia. v.10, n.1, p. $155-76,1980$.

22-OLIVER J., J.E.; LOREN7, M.D. Handbook of veterinary neumologic diagnosis. Philadelphia. W.B.Saunders, 1983.672. p.

23-PALMER, A.C. Other Investigations. In: Palmer, A.C. Introduction to animal neurology. Oxford, Blackwell Scientific Publications, 1976. p. 115-34

24-REIS, J.B.; BEL, A.; REIS FILHO, J.B. Líquido cefalorraquidiano. São Paulo, Sarvier, 1980, 250p.

25-SHERWIN, A.L.; NORRIS. J.W.; BULCKE, J.A. Spinal fluid creatine kinase in neurologic disease. Neurology, Cleveland. v. 19,p.993-9, 1969.

26-SPANO, J.S.; HOERLEIN, B.F. Laboratory examinations. In: Spano. J.S.; Hocrlein. B.F. Canine neurology. Philiddelphia, W.B. Saunders, 1978. p.136-49.

27-VOGEL. J.; RUSSO, E.; SII.VA, M.I. Sobre a determinação da glicose e da uréia no líquido cefalorraquidiano do cảo. Veterinária, Rio de Janeiro, v. 7, n.3, p.14-21. 1953.

28-WILSON, J.W. Clinical application of cerebrospinal fluid creatine phosphokinase determination. Journal of the American Veterinary Medical, v.171, n.2, p.2002. 1977.

29-WILSON, J.W., WILTROUT. S.K. Cerebrospinal fluid cteatine phosphokinase in the nomal dog. American Journal of Veterinary Reswearch, v.37, n.9, p. $1(0) 4$. IOO), 1976.

30-WRIGHT, J.A. Cerebmospinal fluid enzyme estimation in the diagnosis of central nervous system damage in the dog. Veterinary Record. London, v.106, n. 3. p.54-7, 1980

31-ZAR, J.H. Biostatistical analysis. Englewood Cliffs. Prentice-Hall, 1984. 71p.
Recebido para publicação: 10/08/95 Aprovado para publicaçāo: 24/06/96 